\title{
Trade-off study of a variation aware determinate wing assembly against a traditional assembly strategy
}

\author{
Konstantinos Bacharoudis ${ }^{1,}$, Otto Jan Bakker ${ }^{2}$, Atanas Popov ${ }^{1}$ and Svetan Ratchev ${ }^{1}$ \\ ${ }^{1}$ Centre for Aerospace Manufacturing, University of Nottingham, Unit 4, Lenton Lane, Nottingham \\ NG7 2PX, United Kingdom \\ ${ }^{2}$ School of Mechanical, Aerospace and Civil Engineering, University of Manchester, Manchester M13 \\ 9PL, United Kingdom
}

\begin{abstract}
The present study introduces a trade-off framework to evaluate assembly concepts to make informed decisions for aircraft wing structures. This includes (1) the design of the assembly concepts and the estimation of the probability of achieving specific Assembly Key Characteristics, (2) the estimation of the associated costs for each assembly concept using technical cost modelling in combination with Monte Carlo simulation techniques to deal with the uncertainty of the input cost and process parameters and (3) the formulation of the decision matrix to assess the alternative concepts. The suggested trade-off concept can easily be expanded to include weight and performance criteria. Results for a $4 \mathrm{~m}$ generic composite wing indicated that both conventional and determinate assembly can be successfully implemented with high value probabilities to achieve the predetermined assembly key characteristics.
\end{abstract}

\section{Introduction}

It is already known that approximately $70 \%$ of the product cost is committed by decisions made at the early stage of the design [1]. For this reason, there is a need to consider design, manufacturing processes and assembly strategies at the very early stage of the design, adopting principles of concurrent engineering. Tools should be developed to help engineers to make informed decisions and thus to perform trade-off analysis based more on quantitative analytical results rather than just engineering judgement.

One such field of application of trade-off studies is in the selection of the appropriate assembly strategy to build an aircraft wing. The assembly process is accomplished at the final stage of the product development and it has the characteristic to reveal most of the design and manufacturing faults and weaknesses.

In a conventional assembly strategy for aircraft structures, parts are located using fixtures while features on the parts, e.g. surfaces or undersized pilot holes, locate the part with respect to (wrt) the fixture and to the other parts using tack fasteners. In this way, parts are brought together in the desired position according to the predetermined Datum Plan

\footnotetext{
*Corresponding author: konstantinos.bacharoudis@nottingham.ac.uk
} 
(DP) e.g. parts should be at the nominal position, and all the holes in the mating surfaces are drilled, a process called matched drilling. To complete the assembly process, each hole must be deburred, and a fastener should be installed. Although efficient for aerospace applications, this traditional way of assembly exhibits some major drawbacks revealing at the same time opportunities for further improvement. To list some of them, the design, manufacturing and use of fixtures that are expensive structures dedicated to the specific product and the lifetime of the specific project, the matched drilling process that can be complex and expensive when drilling through the thickness of thick hybrid stacks e.g. titanium and carbon fibre reinforced plastics (CFRP) or drilling in awkward positions having limited access and increasing the variability of the process. To deal with those process drawbacks, hole to hole or determinate assembly has been suggested and implemented.

The main characteristic of the determinate assembly is that all the features on the parts are predrilled at the manufacturing stage using accurate CNC machining. This should eliminate the need for expensive fixtures to locate the parts or loading-unloading operation steps to clean and deburr after drilling. However, introducing the concept of the determinate assembly should be carefully considered because of the possibility of hole mismatch between the mating parts. Thus, the adoption of a determinate assembly concept implies that there should be in place a strict dimensional management strategy, increased costs due to tighter manufacturing tolerances compared to the conventional assembly as well as new non-recurring costs due to the possibility of using metrology equipment as well.

Research work on the design of a determinate assembly has been performed in the literature e.g. in [2-4]. Statistical tolerance models were proposed in [2, 3] identifying the minimum fastener diameter that can pass through all the holes in a pattern of holes among mating parts. Furthermore, technical feasibility study was implemented in [3] developing cost models and investigating the effect of the hole misalignment that exist in determinate assembly on the static and fatigue strength properties of the parts. Measurement assisted determinate assembly was further developed in [4].

The present study introduces a trade-off framework to systematically evaluate assembly concepts considering the 1) the design of the assembly using connectivity models and homogeneous transformation matrix (HTM) [5]; 2) the variation propagation using differential transformation matrix (DTM); 3) the cost estimation of the various manufacturing processes implementing process-based cost modelling (PBCM) and Monte Carlo simulation method [6]; and 4) the formulation of the decision matrix [7] to identify the most attractive solution.

Due to limited space and as a first step toward the development of the trade-off framework, in this work, only the assembly models are presented as well as the variation propagation analysis for both traditional and determinate assembly design by estimating the probability of achieving specific assembly key characteristics (AKC). The model performance is demonstrated on a $4 \mathrm{~m}$ composite wing assembly, typical of a wing encountered in the development of new prototype aircraft. The proposed models were further validated using off-the-self computer aided tolerancing tool, 3DCS.

\section{Trade-off methodology}

The suggested methodology includes three major modules. The design of the assembly, the cost estimation and the formulation of the design matrix. Concerning the design of the assembly the steps to be followed are: the identification of the critical AKCs of the product e.g. specific gaps crucial for the identified product key characteristic e.g. aerodynamic performance requirements, the development of a Datum Plan and the Datum Flow Chain (DFC) [5] to deliver each AKC, derivation of feasible assembly sequence, the development 
of mathematical models (assembly model) to describe the assembly using HTM [5] while retrieving information from the available CAD models, for specific DP and DFC, identification of the sources of uncertainties that contribute to the variation of the critical AKC, propagation variation analysis using DTM [5] and simulation techniques e.g. Monte Carlo and finally the estimation of the probability to achieve the critical $\mathrm{AKC}$ which is fed to the decision matrix.

Regarding to the estimation of the assembly costs, technical cost modelling analysis is implemented using PBCM method and Monte Carlo simulation to consider uncertainties in the input parameters [8]. Assembly process planning from the previous assembly design activity in combination with cycle time, resources and cost equations are used to estimate the probability distribution of the recurring and non-recurring costs of the assembly process. The probability not to exceed a specific target cost is evaluated and is fed into the decision matrix.

Well-established multi attribute decision making methods are put in place to evaluate the performance score of every concept e.g. the TOPSIS algorithm.

\subsection{Assembly models}

A typical locating scheme that is encountered in several assembly processes is the 3-2-1 scheme. The 3-2-1 location scheme practically can be achieved with several ways, one of them is to use a mating surface as the primary datum and hole/slot features on the part to restrain all the other remaining degrees of freedom (dof). This is depicted in Figure 1 for part $\mathrm{A}$ and part $\mathrm{B}$. The base of the part $\mathrm{A}$ is in contact to the fixture in at least 3 high points constraining 3 dof. Furthermore, the pin-hole system constraints 2 dof and finally the pinslot system constraints the last dof. For this basic example, the assembly model i.e. the ability to describe the position of any point (and its variation) in the assembly wrt to a coordinate frame of interest e.g. the fixture frame using preliminary information from CAD model is presented in section 2.1.1. The formulas were adopted from [5] and they indicate a $2 \mathrm{D}$ analysis. This means that only simple shapes e.g. plates can be analysed. Herein, the suggested implementation has been improved by considering an intermediate frame that permits for more complex shapes e.g. shells to be analysed while the analysis still is performed on the plane defined by the hole and the slot.

\subsubsection{Conventional assembly}

A way to mathematically describe an assembly is to assume that each part has a base coordinate frame. Mating features on parts each have their own frame. A homogeneous matrix transformation allows to say where each feature is on the part with respect to that part's base frame. An assembly is modelled as a chain of these frames. Chaining all the different coordinate systems by multiplying appropriate transforms $T_{i j}$, an assembly model is obtained. The HTM $T_{i j}$ from frame $j$ to frame $i$ is thus given by

$$
T_{t j}=\left[\begin{array}{cc}
R_{t j} & p_{t j} \\
0 T & 1
\end{array}\right]
$$

Where $R_{i j}$ is the $3 \times 3$ rotation matrix and $p_{i j}$ the $3 \times 1$ translation vector. The inverse transformation is given by

$$
\mathrm{T}_{t t}=\left(\mathrm{T}_{t j}\right)^{-1}=\left[\begin{array}{cc}
R_{t j}^{T} & -R_{t j}^{T} p_{t j} \\
0^{T} & 1
\end{array}\right]
$$


In Figure 1, local frames were assigned for both the part A and the fixture in the CAD model. That is, there is the part frame $(\mathrm{O})$, the intermediate frame on the part $(\mathrm{A})$ that gives the ability to analyse more complex shapes of parts with the suggested methodology, the frame on the hole (a), the frame on the slot (b), the fixture frame (S), the intermediate frame on the fixture (F), the frame on the pin that is mating with the hole (1) and the frame on the pin that is mating with the slot (2). From the CAD model, the following transformation matrices can be extracted: $T_{O A}, T_{O a}, T_{O b}, T_{S F}, T_{S 1}$ and $T_{S 2}$. The main idea is to link the part with the fixture and express the position of any point in the part wrt the frame of the fixture (S). Further, variation will be introduced considering the positional tolerances of the features of the part (hole/slot) or the fixture (pin/pin). The varied position of any point in the part will be expressed through the appropriate transformation wrt the fixture frame. In this way when more than one parts are loaded in the fixture, the dimensional relationships between the parts or the fixture can be studied and analysed in a common frame.

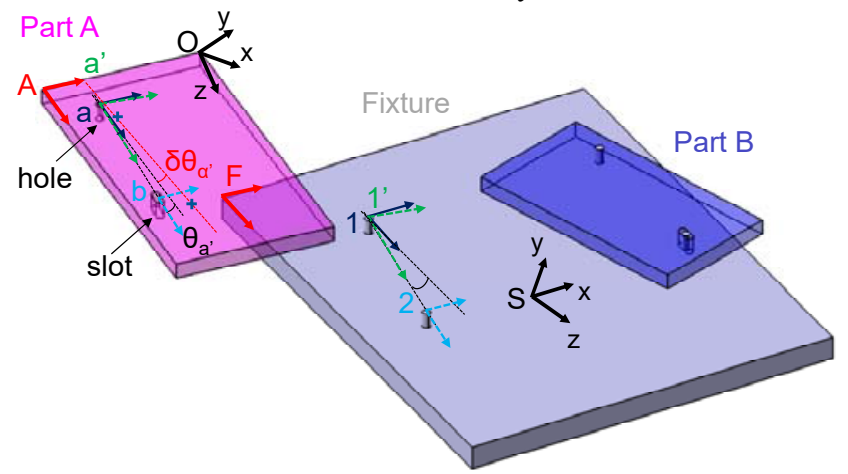

Figure 1: Typical 3-2-1 location of a fixture with two parts (conventional assembly)

The compound features i.e. the hole/slot on the part or pin/pin on the fixture can be described by one additional frame (a' or 1') as depicted in Figure 1. The orientation of this frame wrt a frame of interest can be determined as follow. For the part A, the HTM of the compound feature wrt to frame $\mathrm{A}$ is given by

$$
T_{A a^{l}}=T_{A a^{\prime}} T_{a a^{l}}
$$

where

$$
T_{a a^{\prime}}=\left[\begin{array}{cc}
R_{a a^{\prime}}=R_{a b} & p_{a a^{\prime}}=0 \\
0^{T} & \mathbf{1}
\end{array}\right]
$$

and $R_{a b}=\operatorname{Rot}\left(z, \theta_{a}\right)$ the rotation of the frame a around the $\mathrm{z}$-axis to align with the frame b. The angle $\theta_{a}$, is calculated by geometrical consideration as $\cos \theta_{a^{t}}=\frac{x_{a b}}{\left\|b_{a b}\right\|}$ and $\sin \theta_{a^{t}}=\frac{y_{a b}}{\left\|p_{a b}\right\|}$ where

$$
p_{a b}=p_{A b}-p_{A a}=\left[\begin{array}{l}
x_{a b} \\
y_{a b} \\
z_{a b}
\end{array}\right]
$$

$T_{A a} p_{A b}$ and $p_{A a}$ are calculated from the transformation matrices $T_{O A}, T_{O a}$ and $T_{O b}$ as derived by the CAD model and are given by

$$
\begin{aligned}
& T_{A a}=\left(T_{Q A}\right)^{-1} T_{Q a} \\
& T_{A b}=\left(T_{O A}\right)^{-1} T_{O b}
\end{aligned}
$$

The same formulas hold for the fixture by substituting in Eq. (3)-(7) the subscripts A to F, a to $1, \mathrm{~b}$ to 2 and a' to 1 '. The assembly model for part A, thus, for Figure 1 is given by

$$
T_{S Q}=T_{S E} T_{F 1} T_{1^{\prime} a^{t}}\left(T_{A a^{t}}\right)^{-1}\left(T_{Q A}\right)^{-1}
$$


Where $T_{I^{\prime} a^{\prime}}=I_{4 \times 4}$ for most of the cases. Transform $T_{l^{\prime} a^{\prime}}$ represents the link between the compound frame of the part and the compound frame of the fixture. Eq. (8) can be used to express any point in the part frame to the fixture frame. Similar expressions as Eq. (8) can be developed for any other part loaded to the fixture.

Furthermore, assuming small variations $d p^{a^{t}}$ and $d p^{b^{f}}$ in the position of the hole and the slot wrt the compound frame a' and the frame b respectively due to manufacturing errors, the variation in the angle $\theta_{\alpha}$, is depicted in Figure 1 and can be calculated by

$$
\delta \theta_{a^{t}}=\cos ^{-1}\left(\frac{\operatorname{dot}\left(p_{a_{b b},}^{f} p_{a b}\right)}{\left\|p_{a b}^{f}\right\|\left\|p_{a b}\right\|}\right)
$$

Where $p^{\prime}{ }_{a b}$ the translation from the varied position of the hole to the varied position of the slot depicted in Figure 1. The differential transformation matrix is then given by

$$
D T_{\sigma^{t}}=I_{A \times A}+\left[\begin{array}{cccc}
0 & \delta \theta_{a^{t}} & 0 & d p_{x}^{g^{t}} \\
-\delta \theta_{a^{t}} & 0 & 0 & d p g^{g^{t}} \\
0 & 0 & 0 & 0 \\
0 & 0 & 0 & 0
\end{array}\right]
$$

The same formulas hold for the fixture by substituting in Eq. (9)-(10) the subscripts a to $1, b$ to 2 and a' to 1 '. The assembly model including variation of the features both in the part and the fixture is given by

$$
T_{S O G^{*}}=T_{S E} T_{E 1^{t}} D T_{1^{t}} T_{1^{t} a^{t}}\left(T_{A a^{t}} D T_{a^{t}}\right)^{-1}\left(T_{Q A}\right)^{-1}
$$

Eq. (11) represent the transformation when variation for the features in the part and the fixture are included in the analysis. The random number generation scheme for the $d p^{a^{\prime}}$ and $d p^{b^{\prime}}$ will determine that circular (positional) tolerances are considered in the analysis. This is achieved by sampling the magnitude and the angle of the vector that gives the position of the centre of the hole wrt to the a' frame and then finding the $\mathrm{x}$ and $\mathrm{y}$ components i.e. components of $d p^{a^{\prime}}$. A Rayleigh distribution is assumed for the magnitude and a uniform one for the angle of the positional vector. Monte Carlo simulation is implemented to determine the distribution of the measure of interest.

\subsubsection{Determinate assembly}

Regarding to the determinate assembly, all the holes in the part are pre-drilled in their full size while there is no need of an expensive fixture to give location to the parts. Thus, in Figure $2 \mathrm{a}$, three parts with a pattern of holes are assembled directly by introducing the fasteners. The methodology developed herein calculates the maximum diameter of the fastener that can fit through all the holes. Furthermore, an assembly model is developed according to section 2.1.1 that takes into account the positional tolerance of each hole due to manufacturing error. First step in the methodology is to assign to the CAD model the frames for the mating parts, the intermediate frames and the local frames for every hole as depicted in Figure 2. Second step is to deviate the centre of each hole according to the positional (circular) tolerance assigned in the design and, thus, a hole mismatch is introduced for all the pairs of holes as depicted in Figure 2b. Third step is to express the deviated centres of the holes wrt the part frames. For the fourth step, a searching algorithm is developed to identify the position of the two parts that maximise the diameter of the fastener that can pass through the holes. This is performed by assuming initially that the two parts mate through the first and last hole in the pattern. The HTM is established and is given by

$$
T_{S Q} \operatorname{var}^{2}=T_{S E} T_{F 1^{t}} T_{1^{t} a^{t}} D T_{a^{t}}\left(T_{A a^{t}}\right)^{-1}\left(T_{Q A}\right)^{-1}
$$




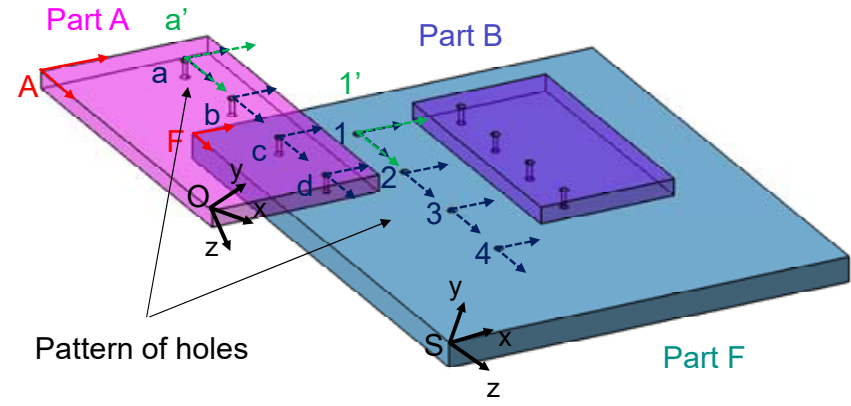

(a)

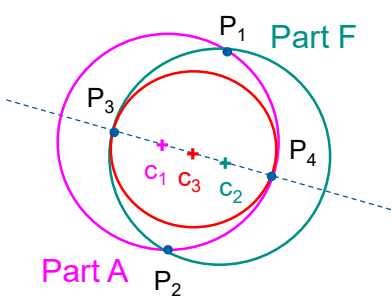

(b)

Figure 2: a) Determinate assembly of 3 parts (b) mismatch of a pair of holes

Where $T_{S F}, T F_{1}, T_{l^{\prime} a^{\prime}}, T_{A a^{\prime}}, T_{O A}$ are HTM calculated by Eq. (1)-(7) and $D T_{a}$, is the DTM that translate the first part relative to the second part wrt the compound frame of the first part, a' and is given

$$
D T_{a^{t}}=I_{4 \times a}+\left[\begin{array}{cccc}
0 & 0 & 0 & d p_{x}^{a^{\prime}} \\
0 & 0 & 0 & d p_{y}^{g^{\prime}} \\
0 & 0 & 0 & 0 \\
0 & 0 & 0 & 0
\end{array}\right]
$$

Thus, for various values of $D T_{a}$, i.e. different relative positions of the mating parts, the maximum diameter of the fastener that can fit through all the pairs of holes can be identified. More specifically, for every value of $D T_{a}$, mating holes takes new positions and the intersection points of the two circles for all the pairs of holes in the pattern are identified. If the intersection points exist i.e. a fastener can pass through all the pair of holes, then the equation of the line that pass from the centres $\left(c_{1}, c_{2}\right)$ of the two holes is calculated, as shown in Figure $2 \mathrm{~b}$. The line intersects the circles in four points, the two inner points $\left(\mathrm{P}_{3}, \mathrm{P}_{4}\right)$ define the diameter of the fastener that can pass through the specific pair of holes. The same procedure is implemented for all the pair of holes in the pattern. The minimum of all the diameters defines the maximum diameter size of the pin that can fit through all the holes. Next step is to find and store the next bigger in size diameter of the fastener that passes through the N-1 holes. At this point, it is known the position of the centres of the two imaginary holes (red circle in Figure 2b) and the maximum diameter of the fastener that can pass through all the pairs of holes in the mating parts. The analysis introduced in Section 2.1.1 can be further performed and an assembly model similar to the one introduced in Eq. (8) can be formulated assuming that the hole and slot feature in this case are the two imaginary holes found from the previous searching algorithm. The derived HTM can be used to express any point in one part wrt the frame of the other including the variation in position of each hole.

\section{Results and Discussion}

\subsection{Case study}

The case study concerns a $4 \mathrm{~m}$ generic wing and is presented in Figure 3 . The simplified wing comprises of two spar beams in "C" shape, one J-nose upper cover, a lower panel and a trailing edge cover. The joining method of the parts is assumed to be mechanical fasteners, while all the laminates are considered monolithic. There are six interface areas 
with rows of holes along the span of the wing (two on the suction side, i.e. upper spar flanges with the J-nose, and four in the pressure side of the wing, i.e. lower spar flanges with the J-nose, lower panel and trailing edge cover).

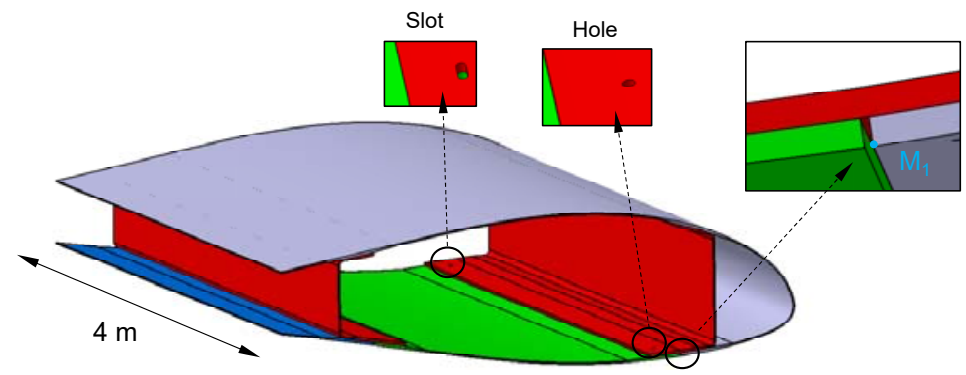

Figure 3: Simplified wing configuration and the AKC under study

One of the most critical requirements concerns the aerodynamic performance of the wing and this is translated in assembly terms that the gap shown in Figure 3 should be less than a target value. Thus, the $\mathrm{AKC}$ in this study is defined as the distance of the point $\mathrm{M}_{1}$ on the J-nose part to the edge of the lower cover, in Figure 3. The specification limit is that this distance should be less than $1 \mathrm{~mm}$. Having identified the AKC, next step is to develop a feasible assembly sequence and establish the Datum Plan.

\subsection{Traditional assembly design}

Firstly, the J-nose with primary datum the outer mould line surface of the panel is loaded in the assembly fixture, followed by the FW spar with primary datum the bag side of the upper flange, and thirdly, the aft spar using the bag side of the upper flange as the primary datum. The lower cover is loaded in the fixture with primary datum the inner mould line surface in contact with the lower flanges of the spars. Finally, the trailing edge cover is mounted in the assembly. For all the parts, the secondary and tertiary datum are pilot holes and slot features e.g. for the FW spar can be seen in Figure 3. Pilot holes of diameter $D_{p}=3 \mathrm{~mm}$ were assumed in the analysis. According to the specific DP, the methodology presented in Section 2 can be applied and the $\mathrm{AKC}$ of interest can be measured. It is obvious that the AKC of interest is affected only by the fixture, the J-nose and the lower cover. Variation is introduced by considering positional tolerances only for the hole on the J-nose and the pin on the fixture that mate with the hole (not shown in Figure 3) and is equal to $T= \pm 0.2 \mathrm{~mm}$. The distribution of the AKC is estimated and presented in Figure 4 by implementing the methodology of Section 2.1.1 as well as using the commercial software 3DCS to validate the outcome of the analysis. The results are in good agreement while the probability of achieving the AKC was estimated $P_{A K C, C}=0.942$.

\subsection{Determinate assembly design}

Regarding to the determinate assembly, parts are bought together and are assembled directly using the pre-drilled full-size holes. Primary datums for all the parts are the same with the ones assumed in the conventional assembly. Full size holes of diameter $D_{p}=5 \mathrm{~mm}$ were assumed in the analysis while for simplicity only 4 holes were assumed in the pattern. The AKC of interest is affected only by the features on the J-nose, the FW spar and the lower cover. Variation is introduced by the positional tolerances of all the holes, $T= \pm 0.2$ $\mathrm{mm}$. The distribution of the AKC is depicted in Figure 4 as well. The probability of achieving the AKC was estimated $P_{A K C, D}=0.961$. From Figure 4, both concepts indicate 
high probability of achieving the key characteristics assuming the same value of positional tolerances in the features in the parts. The maximum diameter fastener identified from the analysis is equal to $D_{f}=4.81 \mathrm{~mm}$ mating with the $\mathrm{J}$ nose and $D_{f}=4.77 \mathrm{~mm}$ for the FW spar.

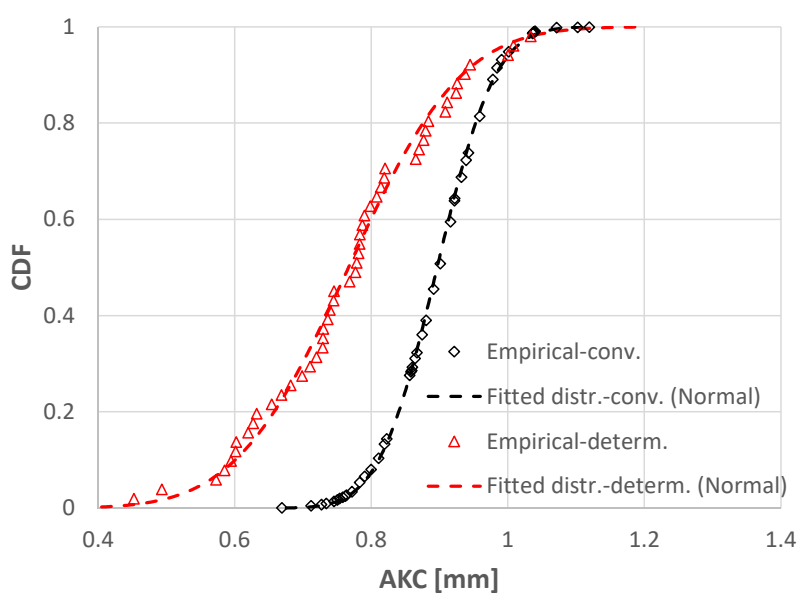

Figure 4: The cumulative distribution function of the AKC

\subsection{Conclusions}

A trade-off framework to systematically evaluate assembly concepts has been developed in this work. Effort was put in the development of the assembly models for a conventional and a determinate assembly strategy for a composite generic wing. The models are developed by using preliminary information from the CAD models. Homogenous transformation matrices were used to build the models while variation was introduced in the form of differential transformation matrices. The analysis indicated that the models are in good agreement with commercial software packages while both concepts indicate high probability to achieve the AKC of interest. The economic analysis of the two solutions should be further implemented and a decision should be made based on both the probability to achieve the AKC and the cost targets for each solution.

\section{References}

1. R. Roy. Decision Engineering Report Series, Cranfield University, (2003)

2. L. Irving, S. Rachev, A. Popov, M. Rafla. SAE Int. J. Aerosp. 7, (2014)

3. J. Bloem. SAE technical paper 2007-01-3926, (2007)

4. T. E. Muelaner, O. C. Martin, P. G. Maropoulos. Procedia CIRP 7, (2013)

5. D. E. Whitney. Mechanical Assemblies: their design, manufacture and role in product development (Oxford University Press, 1998)

6. R. Kirchain, F. R. Field. Encyclopedia of Materials Science \& Engineering, 2, (2000)

7. R. R. Venkata. Decision making in the manufacturing environment: using graph theory and fuzzy multiple attribute decision making methods (Springer, 2007)

8. K. Bacharoudis, T. Turner, A. Popov, S. Ratchev. $8^{\text {th }}$ IPAS 2018, (2018) 